Research Paper

\title{
Transforming growth factor beta (TGF- $\beta$ ) is activated by the CtBP2-p300-AP1 transcriptional complex in chronic renal failure
}

\author{
Ping Zhou ${ }^{1}$, Xiaoxiao Wan ${ }^{1}$, Yan Zou ${ }^{1}$, Zhi Chen ${ }^{2}{ }^{\varpi}$, Aimin Zhong ${ }^{1}$ \\ 1. Department of Nephrology, Jiangxi Provincial People's Hospital Affiliated to Nanchang University, Nanchang 330006, Jiangxi, China. \\ 2. Department of Critical Care Medicine, Jiangxi Provincial People's Hospital Affiliated to Nanchang University, Nanchang 330006, Jiangxi, China.
}

$\square$ Corresponding authors: Dr. Aiming Zhong, Department of Nephrology, Jiangxi Provincial People's Hospital Affiliated to Nanchang University, Nanchang 330006, Jiangxi, China. Tel:+86-791-86897561, Email: aiminzhong@126.com; Dr. Zhi Chen, Department of Critical Care Medicine, Jiangxi Provincial People's Hospital Affiliated to Nanchang University, Nanchang 330006, Jiangxi, China, Tel:+86-791-86896251, Email: zhichen@tongji.edu.cn.

(c) The author(s). This is an open access article distributed under the terms of the Creative Commons Attribution License (https://creativecommons.org/licenses/by/4.0/). See http://ivyspring.com/terms for full terms and conditions.

Received: 2019.07.29; Accepted: 2019.10.15; Published: 2020.01.01

\begin{abstract}
Chronic renal failure (CRF), also known as chronic kidney disease (CKD), is a common renal disorder characterized by gradual kidney dysfunction. Molecular dissection reveals that transforming growth factor beta (TGF- $\beta$ ) plays a central role in the pathogenesis of CRF. However, the mechanism underlying TGF- $\beta$ upregulation has not been demonstrated. Here, we verified that the elevated level of TGF- $\beta$ was associated with the severity of CRF stages and the activation of TGF- $\beta$-mediated signaling in 120 renal biopsies from CRF patients. By analyzing the promoter region of the TGFBI gene, we identified one AP-1 (activator protein 1) and four NF-KB (nuclear factor kappa-light-chain-enhancer of activated $B$ cells) binding sites. Knockdown of two AP-1 subunits (c-Jun and c-FOS) or blockage of AP-1 signaling with two inhibitors T-5224 and SR 11302 could cause the downregulation of TGFBI, whereas knockdown of two NF- $\kappa B$ subunits ( $p 65$ and $p 50$ ) or blockage of NF- $\kappa B$ signaling with two inhibitors TPCAI and BOT-64 could not change the expression of TGFBI. Using mass spectrometry and coimmunoprecipitation analyses, we found that both c-Jun and c-FOS formed a complex with CtBP2 (C-terminal binding protein 2) and histone acetyltransferase $\mathrm{p} 300$. Our in vitro data demonstrated that induction of CtBP2 by recombinant IL-1 $\beta$ (interleukin- 1 beta) led to the upregulation of TGFB I and the activation of TGF- $\beta$ downstream signaling, while knockdown of $C t B P 2$ resulted in the reversed effects. Using chromatin immunoprecipitation assays, we revealed that the CtBP2-p300-API complex specifically bound to the promoter of TGFB and that knockdown or blockage of CtBP2 significantly decreased the occupancies of the p300 and AP-1 subunits. Our results support a model in which the CtBP2-p300-AP1 transcriptional complex activates the expression of TGFBI, increasing its production and extracellular secretion. The secreted TGF- $\beta$ binds to its receptors and initiates downstream signaling.
\end{abstract}

Key words: TGF- $\beta$, CtBP2, p300, c-Jun, c-FOS, chronic renal failure

\section{Introduction}

Chronic renal failure (CRF), also called chronic kidney disease (CKD), is a long-standing and progressive dysfunction of the kidney $[1,2]$. The overall prevalence of CRF in the U.S. adult population is nearly $15 \%[3,4]$, and CRF has become a major public health issue that affects the life quality of individual patients and increases social burden [5-7]. The molecular mechanisms reveal that multiple signaling pathways, such as TGF- $\beta$ (transforming growth factor- $\beta$ )-mediated signaling, Wnt signaling, AKT (AKT serine/threonine kinase, also known as protein kinase B) signaling and NF-кB (nuclear factor 
kappa-light-chain-enhancer of activated B cells) signaling, are activated in the pathogenesis of CRF [8-12]. Of these pathways, TGF- $\beta$-mediated signaling plays a central role [8-12]. Elevated levels of TGF- $\beta$ have been found in CRF patients and in animal models in many laboratories [13-15]. Repression of its coding gene TGFB1 or blockage of TGF- $\beta$ function using its specific antibody can decrease renal scarring [13-15]. Increased TGF- $\beta$ initiates its downstream signaling through binding to a type II TGF-beta receptor (TGFBR2), which recruits and phosphorylates a type I receptor (TGFBR1). TGFBR1 further phosphorylates Smad2 and Smad3, followed by forming a transcriptional complex with Smad4. The accumulations of this complex in the nucleus enable it to regulate the expression of multiple genes, such as CTGF (connective tissue growth factor) and ECM (extracellular matrix) [13-15].

Transcription factors (TFs) play fundamental roles in the regulation of gene expression $[16,17]$. TFs often coordinate with coactivators or corepressors and histone modification enzymes to recruit RNA polymerase II in the promoters of specific genes [16, 17]. Coactivators such as CBP (CREB-binding protein) and EP300 (histone acetyltransferase p300, also known as p300) are directly recruited by TFs to gene promoters to increase gene expression [18, 19]. Corepressors such as CtBPs (C-terminal binding proteins) and NCoR (nuclear receptor corepressor 1) cannot bind to TFs directly but instead interact with repressors to negatively regulate gene expression [20]. Of these corepressors, CtBPs have been well investigated, and they mainly function as oncogenes to repress the expression a variety of genes such as CDH1 (cadherin 1, also known as E-cadherin), Bax and Bim (BCL2 associated X and BCL2 interacting mediator of cell death, respectively), and BRCA1 (breast cancer susceptibility gene 1) [20]. Biochemically, CtBPs directly interact with transcriptional repressors such as HDAC1 (histone deacetylase 1) and HDAC2 or transcriptional activators such as p300 and CBP through a conserved PXDLS motif (where $X$ represents any amino acid) [20]. These repressors and activators are then recruited by TFs such as KLF3 (Kruppel-like factor 3) [21], KLF8 [22], Runx2 (RUNX family transcription factor 2) [23], and TCF4 (Transcription factor 4) [24]. Although CtBP1 and CtBP2 share over 80\% identity in their protein sequences, they do not show significant redundancy in their functions [20]. Intriguingly, in addition to their repressive functions, some studies have also revealed that CtBPs have transcriptional activation ability [25-28]. For example, CtBP2 associates with KLF8 to directly activate the expression level of TIAM1 (T-lymphoma invasion and metastasis-inducing protein 1) to promote human cancer cell migration [25]. In human multidrug resistance (MDR) cancer cell lines, CtBP1 directly activates MDR1 gene expression, thereby increasing P-glycoprotein levels and drug resistance [26]. In gastrointestinal endocrine cells, CtBP1 associates with RAS-responsive element binding protein 1 (RREB1), LSD1 (lysine demethylase 1) and PCAF (P300/CBPassociated factor) to activate NeuroD1-dependent transcription [27]. In the somatic tissue differentiation process, ZNF750 (zinc finger protein 750) physically interacts with KLF4, RCOR1 (REST corepressor 1) and CtBP1/2 to induce the expression of epidermal differentiation genes such as PPL (periplakin), PKP1 (plakophilin 1) and DLX5 (distal-less homeobox 5) [28].

Although the elevated production of TGF- $\beta$ cytokines has been observed in the process of CRF for many years [29], the underlying mechanism of its induction is still obscure. In the present study, we primarily verified the induction of TGF- $\beta$ and the increase in TGFB1 expression at the transcriptional level in kidney biopsies from CRF patients. We then focused our studies on revealing the mechanism of TGFB1 induction in vitro, and our results revealed that the CtBP2-p300-AP1 transcriptional complex was responsible for the upregulation of TGFB1. Our results provide a potential therapeutic strategy of $\mathrm{CRF}$ by targeting individual members of the CtBP2-p300AP1 complex or blocking their interactions.

\section{Materials and methods}

\section{Cell line, cell culture and cell transfection}

A normal human renal cell line, RPTEC/TERT1 OAT3, was purchased from the American Type Culture Collection (ATCC) (Manassas, VA, USA, \#CRL-4031-OAT3). Cells were grown in Dulbecco's modified Eagle's medium (DMEM)/F12 medium (Thermo Fisher Scientific, Waltham, MA, USA, \#12634010) containing 10\% fetal bovine serum (FBS) (Sigma-Aldrich, Shanghai, China, \#F4135) and 1\% penicillin-streptomycin antibiotics (Sigma-Aldrich, \#P4333) at $37^{\circ} \mathrm{C}$. Cells under $80 \%$ confluences were used for transfection to knock down or overexpress genes following a previous protocol [30]. Briefly, siRNAs specifically targeting genes were purchased from Santa Cruz Biotechnology (Dallas, TX, USA), and their catalog numbers were sc-29410 (p65), sc-29407 (p50), sc-29223 (c-Jun), sc-29221 (c-FOS) and sc-37767 (CtBP2). Another CtBP2-siRNA was obtained from ThermoFisher Scientific (\#289298). Plasmids including pCDNA3-2×Flag-c-FOS, pCDNA3-2×Flagc-Jun, pCDNA3-6×Myc-CtBP2, pCDNA3-2×Flag-p300 and pCDNA3- $6 \times$ Myc-p300 were constructed in their 
corresponding empty vectors. For each transfection, $50 \mathrm{pmol}$ of siRNA or $1 \mu \mathrm{g}$ of plasmid was transfected into cells using Lipofectamine 2000 (ThermoFisher Scientific, \#11668019) following the manufacturer's protocol. Cells were then incubated at $37^{\circ} \mathrm{C}$ for $48 \mathrm{~h}$, followed by subjecting to the required experiments.

\section{Blood sample collection and measurement of serum TGF- $\beta$}

Venous blood samples were collected from 24 healthy volunteers (control) and $120 \mathrm{CRF}$ patients who represented 5 different stages $(n=24$ in each stage) according to their glomerular filtration rates (GFR): 1 (GFR $\left.\geq 90 \mathrm{~mL} / \mathrm{min} / 1.73 \mathrm{~m}^{2}\right) ; 2(60<$ GFR $<$ $\left.89 \mathrm{~mL} / \mathrm{min} / 1.73 \mathrm{~m}^{2}\right) ; 3(30<\mathrm{GFR}<59 \mathrm{~mL} / \mathrm{min} / 1.73$ $\left.\mathrm{m}^{2}\right) ; 4\left(15<\mathrm{GFR}<29 \mathrm{~mL} / \mathrm{min} / 1.73 \mathrm{~m}^{2}\right)$; and 5 (GFR < $15 \mathrm{~mL} / \mathrm{min} / 1.73 \mathrm{~m}^{2}$ ) [7]. All patients were diagnosed with CRF and received therapy in the Department of Nephrology, Jiangxi Provincial People's Hospital, during 2012-2016, and their basic characteristics are summarized in Supplementary Table 1. Blood samples were kept in K2EDTA-coated tubes (BD, Franklin Lakes, NJ, USA, \#367835) until use. All participants signed a consent form reviewed and approved by the ethical board of Jiangxi Provincial People's Hospital in China. The serum concentration of TGF- $\beta$ was determined by an ELISA kit (ThermoFisher Scientific, \#BMS249-4).

\section{Renal biopsy collection}

Percutaneous biopsies were collected from 24 renal cell adenocarcinoma patients (TNM stage 1 , used as controls) and 120 CRF patients ( $n=24$ in each stage, and these patients were the same as those for blood sample collection). All participants signed a consent form reviewed and approved by the ethical board of Jiangxi Provincial People's Hospital in China. The primary purpose of renal biopsy collection was to confirm the diagnosis of CRF using immunofluorescence (IF). All patients were given local anesthesia, and the biopsies were collected with disposable, automatic and spring-loaded devices using a 16-gauge needle. After collection, individual biopsies were split into three parts and used for RNA isolation, protein isolation and IF.

\section{RNA isolation and qRT-PCR assay}

Renal biopsies and cultured cells were applied to isolate total RNA using the TRIzol reagent (Sigma-Aldrich, \#T9424) following a protocol provided by the manufacturer. The RNA concentration was quantified using a NanoDrop, and $1.0 \mu \mathrm{g}$ of RNA was utilized to synthesize cDNA using a ProtoScript First Strand cDNA Synthesis Kit (NEB, Beijing, China, \#E6300S). The resulting cDNA was diluted 10-fold and then subjected to qRT-PCR analysis to detect gene expression using the primers listed in Supplementary Table 2. $\beta$-Actin was used as an internal control to normalize the individual gene expression level.

\section{Western blotting and protein level quantification}

Renal biopsies and cultured cells were applied to isolate total protein extracts using $1 \times$ RIPA buffer (Sigma-Aldrich, \#R0278). The protein concentration was determined using a NanoDrop, and $50 \mu \mathrm{g}$ of protein of each sample was loaded into 10\% SDSPAGE gels, followed by transfer to PVDF membranes (Bio-Rad, Shanghai, China, \#1620177). The PVDF membranes were then blocked with $5 \%$ skim milk in $1 \times$ TBST for $1 \mathrm{~h}$, followed by probing with the primary antibodies including anti-Smad2 (Abcam, Shanghai, China, \#ab63576), anti-pSmad2 (Sigma-Aldrich, \#AB3849-I), anti-Smad3 (Abcam, \#ab40854), antipSmad3 (Abcam, \#ab52903), anti-CtBP2 (Abcam, \#ab128871), anti-c-Jun (Santa Cruz Biotechnology, \#sc-166540), anti-c-FOS (Santa Cruz Biotechnology, \#sc-81209), anti-p300 (Sigma-Aldrich, \#P2859), anti-TGF- $\beta$ (Sigma-Aldrich, \#SAB4502954), anti-Flag (Sigma-Aldrich, \#SAB4200071), anti-Myc (SigmaAldrich, \#05419), and anti-GAPDH (Abcam, \#ab181602). After washing 5 times with $1 \times$ TBST, the membrane was probed with secondary antibodies, and the protein signals were detected using an ECL mixture (Sigma-Aldrich, \#GERPN2209) and recorded by a ChemiDoc MP imaging system (Bio-Rad, \#17001402).

\section{Chemical treatments}

Cells were treated with two NF- $\mathrm{BB}$ inhibitors, TPCA1 (5 $\mu$ M, Sigma-Aldrich, \#T1452), and BOT-64 (5 $\mu \mathrm{M}$, Santa Cruz Biotechnology, \#sc-222062), and two AP-1 inhibitors, T-5224 (50 $\mu \mathrm{M}$, GLPBIO, Montclair, CA, USA, \#GC16165) and SR11302 (50 $\mu \mathrm{M}$, Santa Cruz Biotechnology, \#sc-204295), for $4 \mathrm{~h}$ and then stimulated with $50 \mathrm{ng} / \mathrm{mL}$ recombinant IL-1 $\beta$ for $2 \mathrm{~h}$. To block CtBP2-mediated signaling, cells were treated with two CtBP inhibitors, MTOB $(5 \mu \mathrm{M}$, MedKoo Biosciences, Morrisville, NC, USA, \#561414) and NSC95397 (5 $\mu \mathrm{M}$, Sigma-Aldrich, \#N1786). In addition, cells were also only treated with different concentrations $(0,5,50$ and $500 \mathrm{ng} / \mathrm{mL})$ of IL- $1 \beta$. The resulting cells were applied to RNA or protein isolation to detect gene expression or protein level changes.

\section{Immunoprecipitation and mass spectrometry}

Cells expressing pCDNA3-2×Flag-c-FOS or pCDNA3-2×Flag-c-Jun were lysed using $1 \times$ RIPA 
buffer containing $1 \times$ protease inhibitor (SigmaAldrich, \#S8830), followed by centrifuging at 13,000 rpm for $15 \mathrm{~min}$. The supernatant was immunoprecipitated with anti-Flag agarose (Sigma-Aldrich, \#A2220). The purified Flag-c-Jun and Flag-c-FOSassociated complexes were separated on an SDS gel and stained with a Pierce Silver Stain Kit (ThermoFisher Scientific, \#24612). The stained bands were excised and fragmented as described previously [30]. The resulting proteins were analyzed with a $Q$ Exactive $^{\mathrm{TM}}$ HF-X Hybrid Quadrupole-Orbitrap ${ }^{\mathrm{TM}}$ Mass Spectrometer (ThermoFisher Scientific, \#0726042). The obtained data were blasted in the NCBI database using the MASCOT search engine (V.2.3).

\section{Coimmunoprecipitation (Co-IP)}

Cells were transfected with the following combinations of plasmids: pCDNA3- $6 \times \mathrm{Myc}^{+}$ pCDNA3-2×Flag-c-Jun; pCDNA3-6 $\times$ Myc + pCDNA3$2 \times$ Flag-c-FOS; pCDNA3-6 $\times$ Myc-CtBP2+ pCDNA3-2× Flag-c-Jun; $\quad$ CDNA3-6 $\times$ Myc-CtBP2+pCDNA3- $2 \times$ Flag-c-FOS; $\quad$ pCDNA3-6 $\times$ Myc + pCDNA3-2×Flag; pCDNA3-6 $\times$ Myc+pCDNA3-2×Flag-p300; pCDNA3$6 \times$ Myc-CtBP2+pCDNA3-2×Flag; pCDNA3-6 $\times$ MycCtBP2+pCDNA3-2×Flag-p300; $\quad$ pCDNA3-6 $\times$ Myc + pCDNA3-2×Flag-c-Jun; pCDNA3- $6 \times \mathrm{Myc}+$ pCDNA32×Flag-c-FOS; pCDNA3-6×Myc-CtBP2+pCDNA3-2 $\times$ Flag-c-Jun; and pCDNA3-6 $\times$ Myc-CtBP2+pCDNA3$2 \times$ Flag-C-FOS. After incubation at $37^{\circ} \mathrm{C}$ for another 48 $\mathrm{h}$, cells were lysed using $1 \times$ RIPA buffer containing $1 \times$ protease inhibitor. Total cell lysates were centrifuged at $13,000 \mathrm{rpm}$ for $15 \mathrm{~min}$, and the supernatant was immunoprecipitated with anti-Flag agarose and anti-Myc-agarose (Sigma-Aldrich, \#A7470) to pull down proteins. The input and output proteins were detected using western blotting analyses.

\section{Chromatin immunoprecipitation (ChIP) assay}

Cells $\left(5 \times 10^{6}\right)$ were washed twice with PBS buffer and incubated with $1 \%$ formaldehyde at room temperature for $15 \mathrm{~min}$, followed by adding glycine to a final concentration of $125 \mathrm{mM}$ for another $5 \mathrm{~min}$. Cells were further rinsed twice with PBS buffer. The resulting cells were then applied to the ChIP assay using a high sensitivity kit (Abcam, \#ab185913) following a protocol provided by the manufacturer. Antibodies used for immunoprecipitation included anti-CtBP2, anti-c-Jun, anti-c-FOS and mouse IgG (Santa Cruz Biotechnology, \#sc-2025). The relative enrichment of promoters was determined by qRT-PCR using the primers listed in Supplementary Table 3.

\section{Statistical analysis}

All experiments in this study were independently repeated in triplicate. Data are presented as the mean \pm SEM from a representative experiment. Statistical analyses were performed using SPSS version 22 software. The significance levels were set at $P<0.05\left(^{*}\right), P<0.01\left(^{* *}\right)$ and $P<0.001\left(^{* * *}\right)$.

\section{Results}

\section{TGF- $\beta$ levels were elevated, and TGF- $\beta$ - mediated signaling was activated in CRF patients}

Although many publications have reported an increase in TGF- $\beta$ levels in CRF patients $[13,29]$, it is still unclear whether TGF- $\beta$ levels are associated with the severity of CRF stages. To verify this hypothesis, we measured circulating TGF- $\beta$ levels in serum from 24 healthy volunteers and 120 CRF patients who underwent 5 different stages (from stage 1 to $5, n=24$ in each stage). Our results showed that TGF- $\beta$ levels in CRF patients were significantly increased compared to controls (Figure 1A). More importantly, the circulating concentration of TGF- $\beta$ was positively associated with the severity of CRF stages, that is, the higher the TGF- $\beta$ concentration, the more severe is the CRF (Figure 1A). Then, we combined the TGF- $\beta$ concentration in individual CRF patients and compared them with healthy controls. Consistently, we also found a remarkable increase in TGF- $\beta$ levels in CRF patients (Figure 1B). The increased level of TGF- $\beta$ implied that its encoded gene TGFB1 might be upregulated in CRF patients. To test this hypothesis, we isolated total RNA from renal biopsies of CRF patients and examined TGFB1 mRNA levels in these clinical samples. Consistent with its circulating level, we also found that TGFB1 mRNA levels were gradually increased with the severity of the CRF stages (Figure 1C). The combined data also indicated that TGFB1 mRNA levels were dramatically increased in CRF patients (Figure 1D). It is well known that the accumulation of TGF- $\beta$ can activate its downstream signaling, causing an increase in Smad2 and Smad3 phosphorylation levels. Thus, we also examined the total and phosphorylated levels of Smad2 and Smad3 in one control biopsy and 5 CRF biopsies at different stages. As shown in Figures $1 \mathrm{E}$ and $1 \mathrm{~F}$, the phosphorylated levels of Smad2 and Smad3, but not their total protein levels, were gradually increased with the severity of CRF stages. These results clearly indicated that the TGF- $\beta$ level was elevated and that TGF- $\beta$-mediated signaling was activated in CRF patients. 


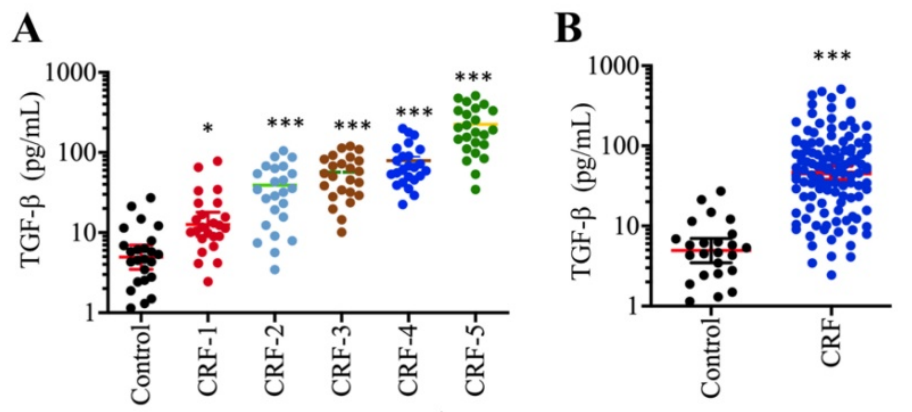

C

D

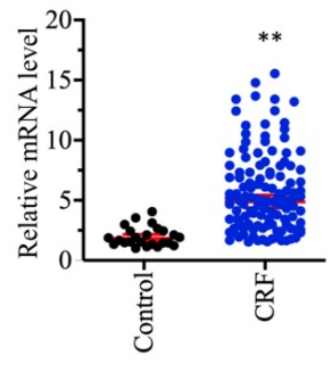

E

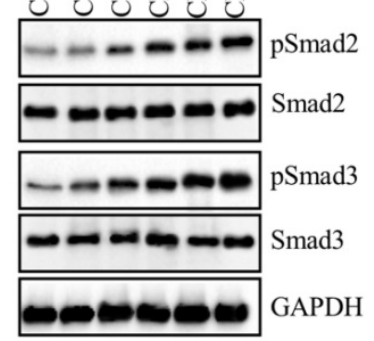

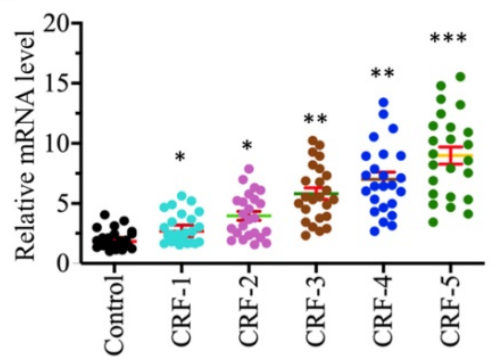

F

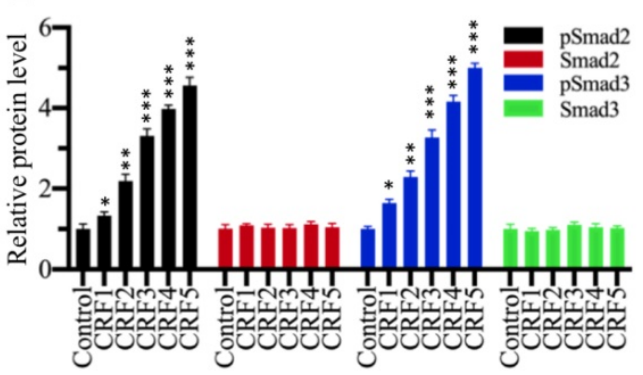

Figure 1. The serum concentration of TGF- $\beta$ was elevated and TGF- $\beta$ signaling was activated in CRF patients (A) Serum concentrations of TGF- $\beta$ were associated with the CRF stage. Circulating levels of TGF- $\beta$ were measured in serum samples obtained from healthy controls ( $\mathrm{n}=24)$ and $\mathrm{CRF}$ patients at 5 different stages $(1-5, n=24$ in each stage) $* P<0.05$ and $* * * P<0.001$. (B) Serum concentrations of TGF- $\beta$ were elevated in CRF patients. Circulating levels of TGF- $\beta$ in CRF patients $(n=120)$ were combined and compared with levels in controls. $* * * P<0.001$. (C) The relative expression level of TGFBI was associated with the CRF stage. The qRT-PCR analyses were performed to measure TGFBI mRNA levels in renal biopsies from renal cell adenocarcinoma patients (TNM stage 1, control) and $120 \mathrm{CRF}$ patients representing 5 different stages $(n=24$ in each stage). $* P<0.05, * * P<0.01$ and $* * * P<0.001$. (D) The relative expression level of TGFBI was elevated in CRF patients. The relative expression levels of TGFBI in CRF patients $(n=120)$ were combined and compared with levels in controls. $* * P<0.01$. (E and F) TGF- $\beta$ signaling was activated in CRF patients. (E) Western blotting was performed to measure pSmad2, Smad2, pSmad3 and Smad3 in renal biopsies from one renal cell adenocarcinoma patient (TNM stage 1 , control) and 5 CRF patients ( $n=1$ in each CRF stage). GAPDH was used as a loading control. (F) The relative protein levels in (E) were quantified and normalized to GAPDH. $* P<0.05$, $* * P<0.01$ and $* * * P<0.001$.

\section{AP-1 specifically regulated the expression of TGFB I in vitro}

To explore the underlying mechanism of TGFB1 induction in CRF patients, we primarily analyzed the TF binding sites in the promoter of TGFB1. We selected a 1500-bp length of the promoter and found four NF-кB sites [-189-(-198), -629-(-)638, -779-(-)788 and -881-(-)890] and one AP-1 site [-337-(-343)] (Figure $2 \mathrm{~A})$. The mammalian genome encodes five NF- $\mathrm{BB}$ members, including p65/RELA, RELB, c-REL, p50/NFKB1 and p52/NFKB2, and two AP-1 subunits, including c-Jun and c-FOS. Since TGFB1 mRNA levels were significantly induced in CRF patients, we speculated that NF- $\mathrm{KB}$ and AP-1 might be also upregulated in the same samples if they could directly regulate the expression of TGFB1. Thus, we measured the mRNA levels of two members of NF- $\mathrm{B}$, p 65 and p50, and two subunits of AP-1 in CRF renal samples. Interestingly, we found that both the NF- $\mathrm{BB}$ and AP-1 subunits were slightly upregulated in CRF patients compared to controls (Figures 2B-2E). To further examine whether NF- $\mathrm{KB}$ or AP-1 was directly involved in the regulation of TGFB1 expression, we knocked down p65, p50, c-Jun and c-FOS in a human RPTEC-TERT1 OAT3 cell line obtained from normal kidney. After verification of their successful knockdown (Supplementary Figures 1A-1D), we examined TGFB1 mRNA levels in these cells. Our results indicated that knockdown of AP-1 subunits but not NF-KB subunits significantly repressed the expression of TGFB1 (Figure 2F). We also used recombinant IL-1 $\beta$ to stimulate these knockdown cells. The results showed that the expression of TGFB1 in sip65- and sip50-cells after IL-1 $\beta$ stimulation was significantly induced to a comparable level as that in control cells (Figure 2F). However, knockdown of c-Jun and c-FOS impaired the induction of TGFB1 with IL-1 $\beta$ stimulation (Figure 2F), which was significantly different from that in control and sip65and sip50-cells (Figure 2F). In addition, we also treated RPTEC-TERT1 OAT3 cells with two inhibitors of NF- $\kappa$ B signaling, TPCA-1 and BOT-64, and two AP-1 inhibitors, T-5224 and SR11302, respectively. Consistent with the above knockdown results, we also observed that only AP-1 inhibitors but not NF-кB inhibitors can decrease the expression of TGFB1, and IL-1 $\beta$ stimulation can only slightly induce TGFB1 levels in cells previously treated with AP-1 inhibitors (Figure 2G). These results suggested that AP-1 was able to regulate the expression of TGFB1 in vitro. 

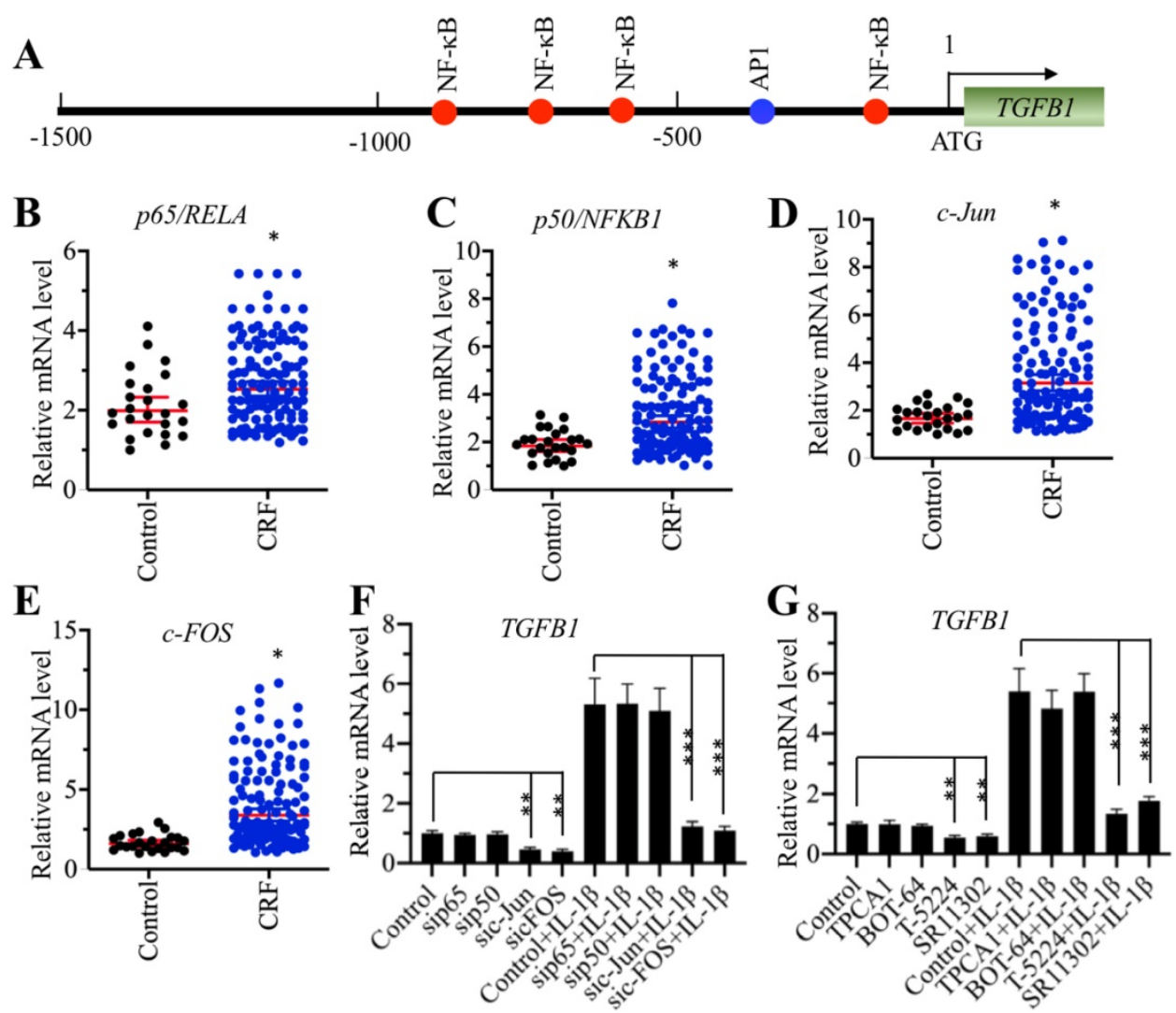

Figure 2. Knockdown of AP-1 subunits caused the downregulation of TGFBI. (A) The promoter of TGFBI contained one AP-1 and four NF- $k B$ binding sites. A 1500 bp-length TGFBI promoter was selected and applied to predict transcription factor binding sites from a website (http://alggen.lsi.upc.es). The binding positions of one AP-1 and four NF-kB binding sites are indicated. (B and C) The mRNA levels of p65/RelA and p50/NFKBI were upregulated in CRF patients. The same RNA samples used in Figure $1 C$ were applied to measure the mRNA levels of $p 65$ (B) and $p 50$ (C). $* P<0.05$. (D and E) The c-jun and $c$-FOS mRNA levels were upregulated in CRF patients. The same RNA samples used in Figure $1 C$ were applied to measure the mRNA levels of $C-J u n(D)$ and $c-F O S(E)$. $* P<0.05$. (F) The effects of knockdown of NF-kB and AP-1 subunits on the expression of TGFBI. Cells with knockdown of p65, p50, c-Jun or c-FOS were treated with or without $50 \mathrm{ng} / \mathrm{mL}$ recombinant IL-1 $\beta$, followed by RNA isolation and qRT-PCR analyses to measure TGFBI mRNA levels. **P $<0.01$ and $* * * P<0.001$. (G) The effects of blocking the NF- $\mathrm{KB}$ and AP-1 signaling pathways on the expression of TGFBI. Cells were primarily treated with two NF- $\mathrm{KB}$ signaling inhibitors, TPCA-1 and BOT-64, and two AP-1 signaling inhibitors, T-5224 and SR 1 1302, respectively, followed by further treatment with or without $50 \mathrm{ng} / \mathrm{mL}$ IL-1 $\beta$. After RNA isolation, qRT-PCR analyses were performed to measure TGFBI mRNA levels. $* * P<0.01$ and $* * * P<0.001$

\section{AP-1 formed a transcriptional complex with p300 and CtBP2}

TFs recruit many other proteins to regulate gene expression [16, 17]. To identify the members of the AP-1-associated transcriptional complex, we transfected RPTEC-TERT1 OAT3 cells with pCDNA3$2 \times$ Flag-c-FOS and pCDNA3-2×Flag-c-Jun. After immunoprecipitation with anti-Flag agarose, the Flag-c-FOS and Flag-c-Jun-associated protein complexes were applied to sliver staining (Figure 3A) followed by mass spectrometry analysis to identify proteins in these two complexes. We identified 35 proteins in the Flag-c-FOS-associated complex (Supplementary Table 4) and 29 proteins in the Flag-c-Jun-associated complex (Supplementary Table 5). Comparing these two protein lists, we found that p300 and CtBP2 overlapped (Supplementary Table 4 and 5). This result, together with previous publications [23, 30,31], suggested that these two proteins had high possibilities of being recruited by
c-Jun and c-FOS, thereby forming two transcriptional complexes. To verify this hypothesis, we primarily examined the in vivo association of CtBP2, p300 and AP-1 subunits. Accordingly, we determined the existence of CtBP2 and p300 in the immunoprecipitated protein materials used for mass spectrometry analysis. The results showed that both CtBP2 and p300 can be detected in both Flag-c-FOS and Flag-c-Jun-associated complexes (Figure 3B). Then, we performed Co-IP assays to examine their direct interactions. To determine if CtBP2 can directly interact with c-FOS and c-Jun, we cotransfected the following combinations of plasmids including pCDNA3-6×Myc+pCDNA3-2×Flag-c-Jun; pCDNA3$6 \times \mathrm{Myc}+$ pCDNA3-2 $\times$ Flag-c-FOS; pCDNA3-6 $\times$ MycCtBP2+pCDNA3-2×Flag-c-Jun; and pCDNA3-6×MycCtBP2+pCDNA3-2×Flag-c-FOS into RPTEC-TERT1 OAT3 cells. The Co-IP results suggested that CtBP2 cannot directly interact with c-Jun or c-FOS (Figure 3C). To determine if CtBP2 directly interacted with p300, we cotransfected the following combinations of 
plasmids including pCDNA3-6 $\times \mathrm{Myc}+\mathrm{pCDNA3}-2 \times$ Flag; $\quad$ pCDNA3- $6 \times$ Myc + pCDNA3-2 $\times$ Flag-p300; pCDNA3-6×Myc-CtBP2+pCDNA3-2×Flag; and pCDNA3-6×Myc-CtBP2 + pCDNA3-2×Flag-p300 into RPTEC-TERT1 OAT3 cells. The Co-IP results suggested that CtBP2 could directly interact p300 (Figure 3D). Following this, we determined the direct interactions of p300-c-Jun and p300-c-FOS using cells

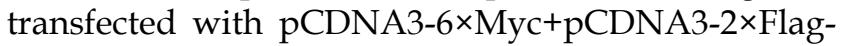
c-Jun; pCDNA3- $6 \times$ Myc + pCDNA3-2×Flag-c-FOS; pCDNA3-6 $\times$ Myc-p300+pCDNA3-2 $\times$ Flag-c-Jun; and pCDNA3-6×Myc-p300+pCDNA3-2×Flag-c-FOS. The results showed that $\mathrm{p} 300$ directly interact with c-Jun and c-FOS (Figure 3E). Thus, we concluded that AP-1 subunits directly interacted with p300, which further
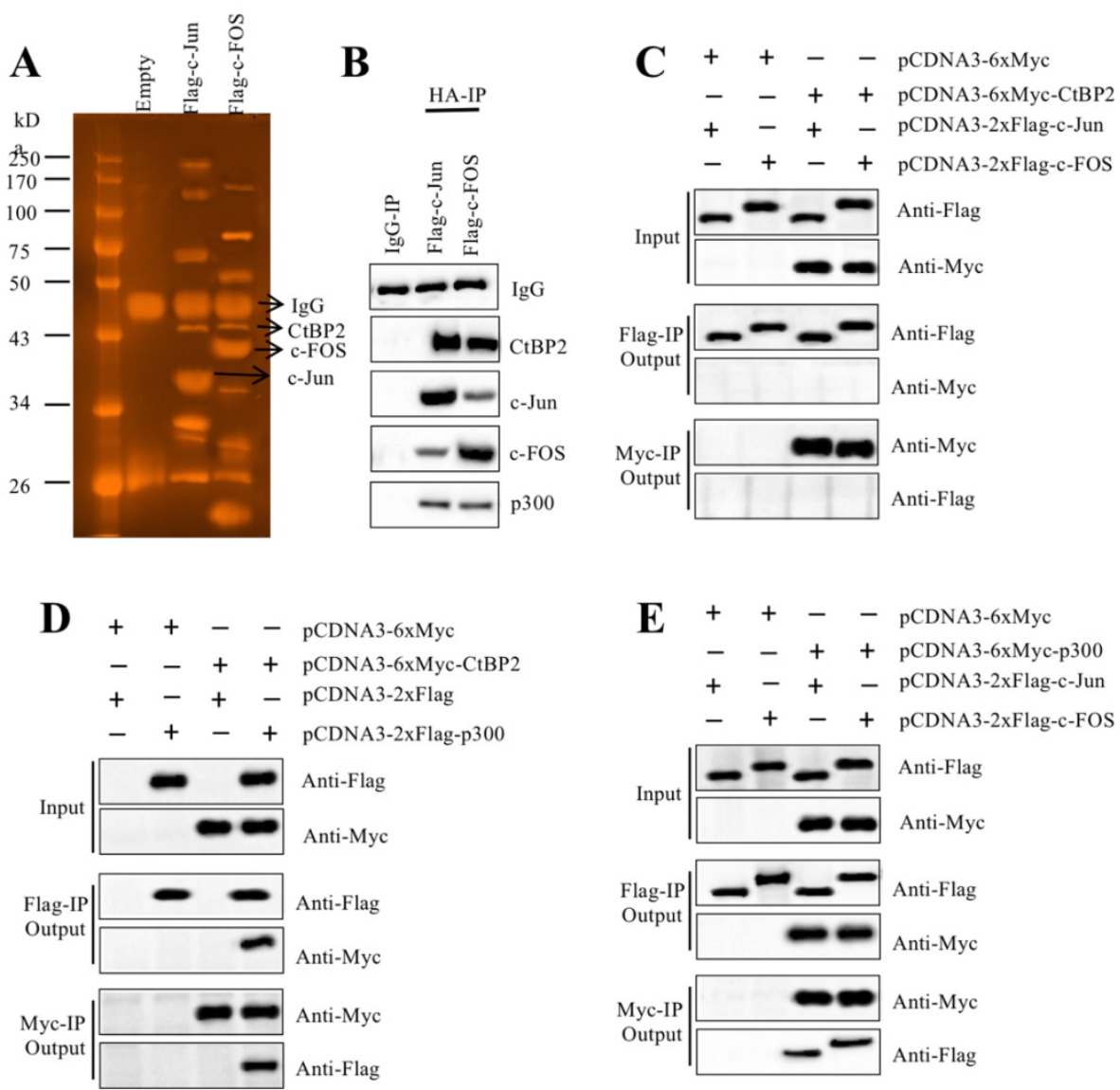

Figure 3. CtBP2 formed transcriptional complexes with p300 and c-Jun or c-FOS. (A) In vivo pull-down of the Flag-c-Jun- and c-FOS-associated

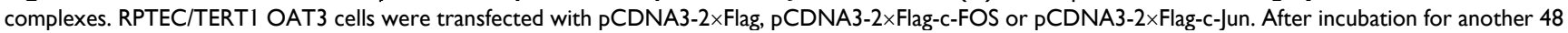
$\mathrm{h}$, cells were subjected to immunoprecipitation analysis. The purified protein complexes were loaded onto SDS-PAGE gels, and protein bands were visualized using sliver staining. The lgG, c-Jun, c-FOS and CtBP2 bands are indicated. (B) Verification of the association of CtBP2, p300 and c-Jun or c-FOS in vivo. Protein samples used for mass spectrometry were applied to western blotting analyses to verify the association of CtBP2, p300 and c-Jun or c-FOS. (C) CtBP2 could not interact directly with c-Jun or c-FOS. The RPTEC/TERTI OAT3 cells were transfected with the following combinations of plasmids: $p C D N A 3-6 \times$ Myc $+p C D N A 3-2 \times F l a g-c-J u n$;

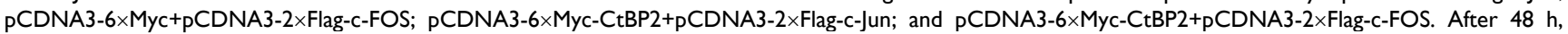
cells were subjected to immunoprecipitation analysis using anti-Flag agarose and anti-Myc agarose, respectively. The pull-down products were used to determine protein interactions by probing with the antibodies indicated in the figures. (D) CtBP2 directly interacts with $p 300$. The RPTEC/TERT1 OAT3 cells were transfected

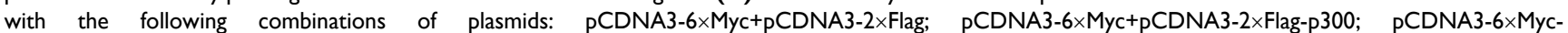

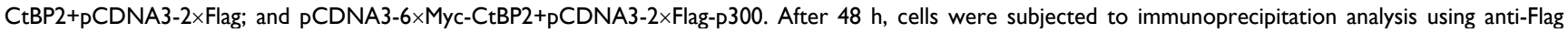
agarose and anti-Myc agarose, respectively. The pull-down products were used to determine protein interactions by probing with the antibodies indicated in the figures. (E) p300 directly interacted with c-Jun or c-FOS. The RPTEC/TERT1 OAT3 cells were transfected with the following combinations of plasmids:

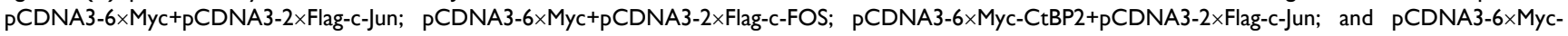
CtBP2+pCDNA3-2×Flag-c-FOS. After $48 \mathrm{~h}$, cells were subjected to immunoprecipitation analysis using anti-Flag agarose and anti-Myc agarose, respectively. The pull-down products were used to determine protein interactions by probing with the antibodies indicated in the figures. 

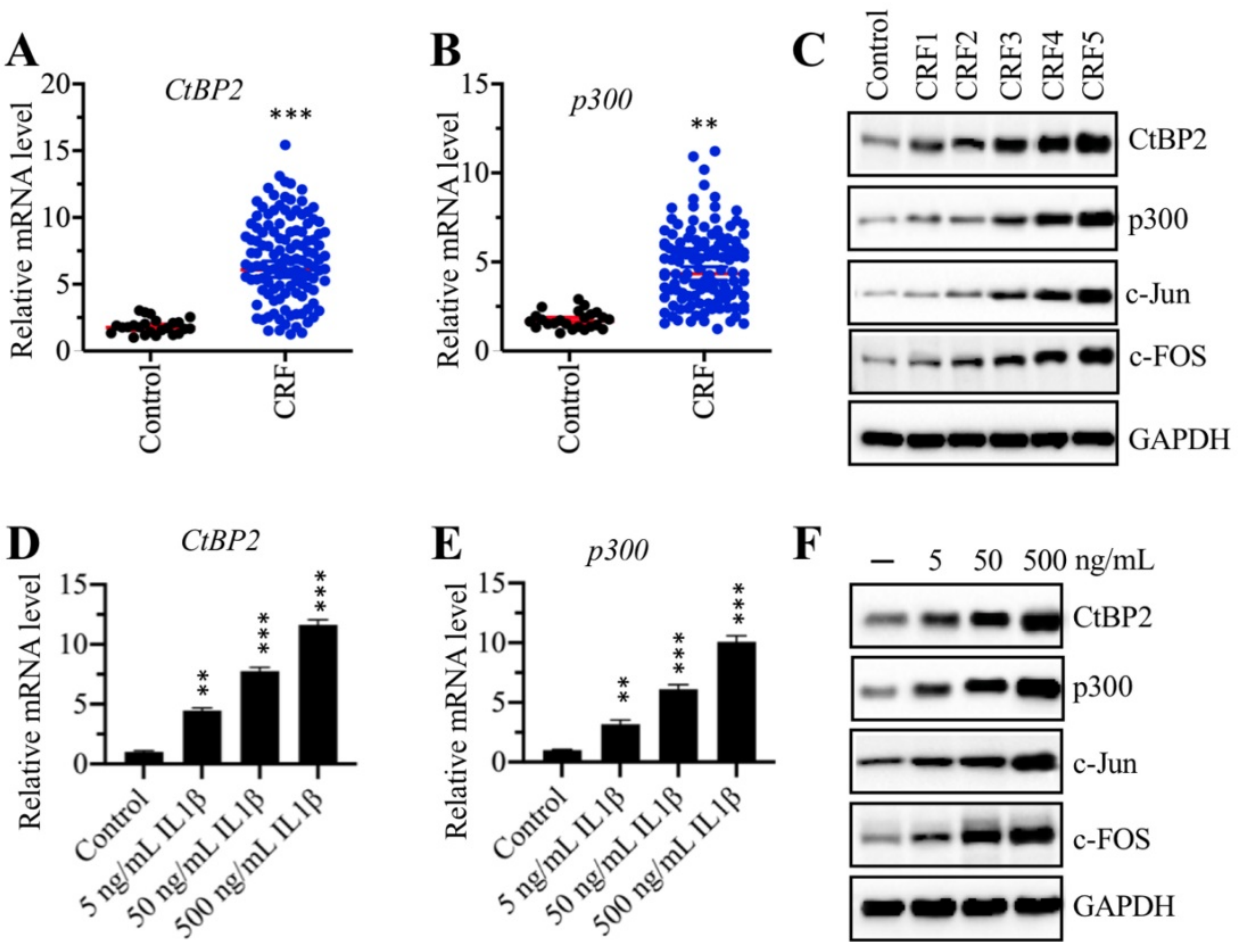

Figure 4. The CtBP2-p300-API transcriptional complexes were upregulated in CRF patients. (A) The CtBP2 mRNA level was elevated in CRF patients. The RNA samples used in Figure $1 C$ were subjected to measurement of the mRNA level of $C t B P 2$. ${ }^{*} * * P<0.001$. (B) The $p 300$ mRNA level was elevated in CRF patients. The RNA samples used in Figure $1 C$ were subjected to measurement of the mRNA level of $p 300$. $* * P<0.01$. (C) The protein levels of CtBP2-p300-AP1 components increased with the severity of CRF stage. The protein samples used in Figure 1E were used to determine the levels of CtBP2, p300, c-Jun and c-FOS. GAPDH was used as a loading control. (D) IL-1 $\beta$ stimulation induced the expression of CtBP2. Cells were treated with different concentrations of IL-1 $\beta$ including 0 , 5,50 and $500 \mathrm{ng} / \mathrm{mL}$ for $2 \mathrm{~h}$, followed by RNA isolation and qRT-PCR analyses to examine CtBP2 mRNA levels. $* * P<0.01$ and $* * * P<0.001$. (E) IL-1 $\beta$ stimulation induced the expression of $p 300$. RNA samples used in (D) were subjected to qRT-PCR analyses to examine the $p 300$ mRNA level. $* * P<0.01$ and $* * * P<0.001$. (F) The protein levels of CtBP2-p300-API components were increased with IL-1 $\beta$ stimulation. Cells used in (D) were subjected to protein isolation to determine levels of CtBP2, p300, c-Jun and c-FOS. GAPDH was used as a loading control.

Our results indicated that both CtBP2 and p300, as well as c-FOS and c-Jun, gradually increased with the severity of the CRF stage (Figure 4C and Supplementary Figure 2A). Recombinant IL-1 $\beta$ stimulation could result in the induction of TGFB1; thus, we next evaluated the effect of IL-1 $\beta$ stimulation on the mRNA and protein levels of CtBP2 and p300. Accordingly, we treated RPTEC-TERT1 OAT3 cells with different concentrations $(0,5,50$ and $500 \mathrm{ng} / \mathrm{mL})$ of IL-1 $\beta$. The qRT-PCR analysis results showed that both CtBP2 and $p 300$ mRNA levels were significantly increased after IL-1 $\beta$ stimulation in a dose-dependent manner (Figures 4D and 4E). Consistent with their mRNA levels, we also observed that the protein levels of CtBP2, p300, c-Jun and c-FOS were induced with IL-1 $\beta$ treatment, and their inductions were dependent on the dose of IL-1 $\beta$ (Figure $4 \mathrm{D}$ and Supplementary Figure 2B).

\section{CtBP2 served as a coactivator in the regulation of TGFBI expression}

The same expression patterns of the CtBP2, p300, and $A P-1$ subunits and TGFB1 in CRF biopsies and in IL-1 $\beta$-treated cells implied that CtBP2 might not serve as a corepressor but instead as a coactivator in the regulation of TGFB1 expression. To verify this hypothesis, we knocked down CtBP2 with two specific siRNAs in RPTEC-TERT1 OAT3 cells to generate siCtBP2-1 and siCtBP2-2 cells, followed by treatment with or without recombinant IL-1 $1 \beta$. After verifying the successful knockdown of $C t B P 2$ and induction by IL-1 $\beta$ (Figure $5 \mathrm{~A}$ ), we evaluated the expression of TGFB1. The results showed that knockdown of $C t B P 2$ dramatically decreased the expression of TGFB1, and the induction of TGFB1 in the treatment of IL-1 $\beta$ was also dependent on CtBP2 because IL-1 $\beta$ stimulation only slightly increased TGFB1 expression in CtBP2-knockdown cells (Figure $5 B)$. Since changes in $C t B P 2$ expression could affect TGFB1 expression, it should also affect TGF- $\beta$ downstream signaling. For this purpose, we also determined the protein levels of CtBP2, TGF- $\beta$, pSmad 2 and Smad2 in CtBP2-knockdown cells as well as in cells treated with or without IL-1 $\beta$. Consistent with their mRNA levels, we also found a significant decrease in TGF- $\beta$ protein levels after CtBP2 knockdown, and IL-1 $\beta$ only slightly induced the expression of TGF- $\beta$ (Figure 5C and Supplementary Figure $3 \mathrm{~A})$. As expected, CtBP2 knockdown caused a 
block in TGF- $\beta$ downstream signaling because we observed a significant decrease in the pSmad2 level (Figure 5C and Supplementary Figure 3A). To further confirm our observation, we also treated RPTEC-TERT1 OAT3 cells with two CtBP inhibitors including MTOB and NSC95397, which have been reported to block CtBP function and CtBP-mediated signaling in multiple publications [20]. Similarly, we also examined the mRNA levels of CtBP2 and TGFB1 with the treatment of these two inhibitors. Our results showed that both MTOB and NSC95397 treatments could not change CtBP2 mRNA levels; however, they significantly decreased the expression of TGFB1 (Figures 5D and 5E). In the treatments with MTOB or NSC95397, IL-1 $\beta$ stimulation still markedly induced the expression of $C A B P 2$, but not TGFB1 (Figures 5D and $5 \mathrm{E}$ ). Similarly, we also examined the protein levels of CtBP2, TGF- $\beta$, pSmad2 and Smad2 in cells treated with MTOB and NSC95397, as well as in the condition of IL-1 $\beta$ stimulation. Consistently, we found that both MTOB and NSC95397 treatments significantly decreased TGF- $\beta$ and pSmad 2 levels, and IL-1 $\beta$ stimulation only slightly increased their expression (Figure 5F and Supplementary Figure 3B). These results supported that $\mathrm{CtBP} 2$ functioned as a coactivator in the regulation of TGFB1 expression and TGF- $\beta$ downstream signaling.

\section{The CtBP2-p300-API transcriptional complex specifically bound to the promoter of TGFBI}

Our above results suggested that the CtBP2p300-AP1 complex activated the expression of TGFB1. To further verify the activation role of CtBP2 and determine whether this activation occurred through the direct binding of the CtBP2-p300-AP1 complex to the promoter of TGFB1, we primarily evaluated whether CtBP2 knockdown affected the occupancies of p300, c-Jun and c-FOS in the promoter of TGFB1. Accordingly, we performed ChIP assays in CtBP2-knockdown cells using anti-CtBP2, anti-p300, anti-c-Jun and anti-c-FOS for immunoprecipitation. The results showed that the occupancies of p300, c-Jun
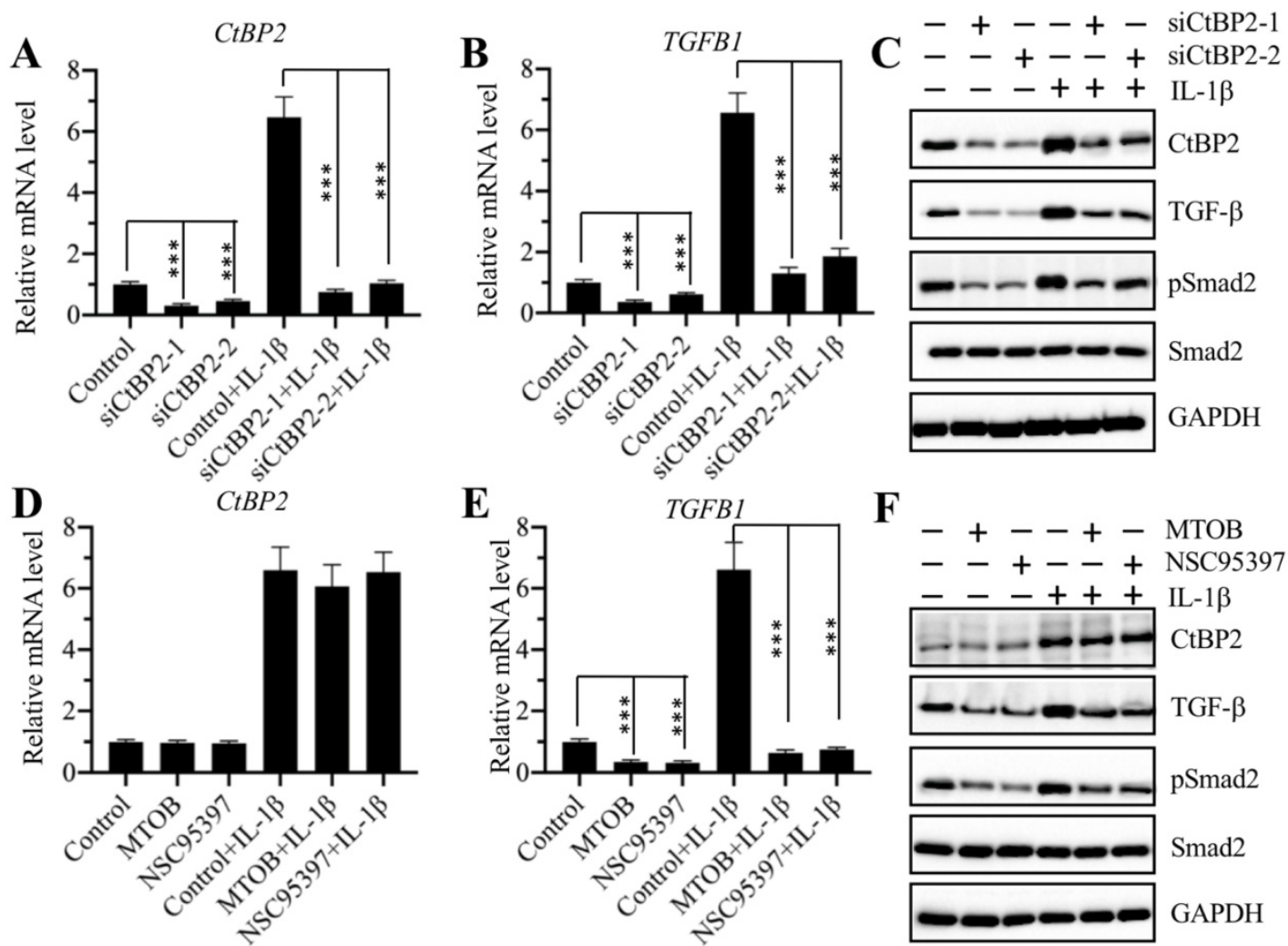

Figure 5. Knockdown of CtBP2 caused the repression of TGFBI. (A) The CtBP2 mRNA level. The RPTEC/TERT1 OAT3 cells were transfected with two independent siRNAs of CtBP2. After incubation for another $48 \mathrm{~h}$, cells were further treated with or without $50 \mathrm{ng} / \mathrm{mL} \mathrm{IL}-1 \beta$ for $2 \mathrm{~h}$, followed by RNA isolation and qRT-PCR analysis to measure CtBP2 mRNA levels. *** $<0.001$. (B) The TGFBI mRNA level. RNA samples used in (A) were applied to $q R T-P C R$ analysis to measure TGFBI mRNA levels. $* * P<0.01$ and $* * * P<0.001$. (C) Knockdown of $C t B P 2$ inhibited TGF- $\beta$ signaling. Cells used in (A) were subjected to protein isolation and western blotting to examine the protein levels of CtBP2, TGF- $\beta$, pSmad2, Smad2 and GAPDH. (D) Treatments with CtBP inhibitors could not change the mRNA level of CtBP2. The RPTEC/TERT1 OAT3 cells were treated with $5 \mu$ M MTOB or NSC 95397 for 4 h. Cells were then further treated with or without 50 $\mathrm{ng} / \mathrm{mL}$ IL- $1 \beta$ for $2 \mathrm{~h}$. The resulting cells were subjected to RNA isolation and qRT-PCR analysis to measure CtBP2 mRNA levels. (E) Treatments with CtBP inhibitors significantly repressed TGFBI mRNA levels. RNA samples used in (D) were applied to qRT-PCR analysis to measure TGFBI mRNA levels. ***P < 0.001 . (F) Treatments with CtBP inhibitors inhibited TGF- $\beta$ signaling. Cells used in (D) were subjected to protein isolation and western blotting to examine the protein levels of CtBP2, TGF- $\beta$, pSmad2, Smad2 and GAPDH. 
and c-FOS in the promoter of TGFB1 were significantly decreased with CtBP2 knockdown (Figure 6A). IL-1 $\beta$ stimulation significantly increased the occupancies of p300 ( 5-fold), c-Jun ( 6-fold) and c-FOS ( 7-fold) in control cells, while it only slightly increased $(\sim 1-$ fold $)$ the occupancies of these proteins in CtBP2-knockdown cells (Figure 6A). In addition, we also performed the same ChIP assays in cells treated with CtBP inhibitors. Similar to the results in CtBP2-knockdown cells, we also observed a significant decrease ( 0.3-0.5-fold) in p300, c-Jun and c-FOS occupancies in cells treated with MTOB or NSC95397 (Figure 6B). Combined treatment with CtBP inhibitors and IL-1 $\beta$ stimulation also only slightly increased the occupancies of p300, c-Jun and c-FOS (Figure 6B). These results suggested that CtBP2 acted as an activator to induce the occupancy of p300-AP1 in the promoter of TGFB1.

\section{Discussion}

Although TGF- $\beta$ induction is recognized as a hallmark of CRF [13-15, 29, 31], little is known about whether its concentration is associated with the severity of the CRF stages. Importantly, the molecular mechanism of TGF- $\beta$ induction is still unknown. To solve these two questions, we primarily measured serum TGF- $\beta$ concentrations in a total of 120 CRF patients at different CRF stages and revealed that the TGF- $\beta$ concentration was associated with the severity of CRF stages (Figure 1A). This result suggested that the serum TGF- $\beta$ concentration might be used as a marker for the diagnosis of CRF. Following this, we used multiple approaches, such as bioinformatics, immunoprecipitation, mass spectrometry, Co-IP and ChIP assays, to identify and verify that the CtBP2-p300-AP1 complex was responsible for the upregulation of TGFB1. To our knowledge, this is the first identification of the activating role of CtBP2 in inflammatory diseases.
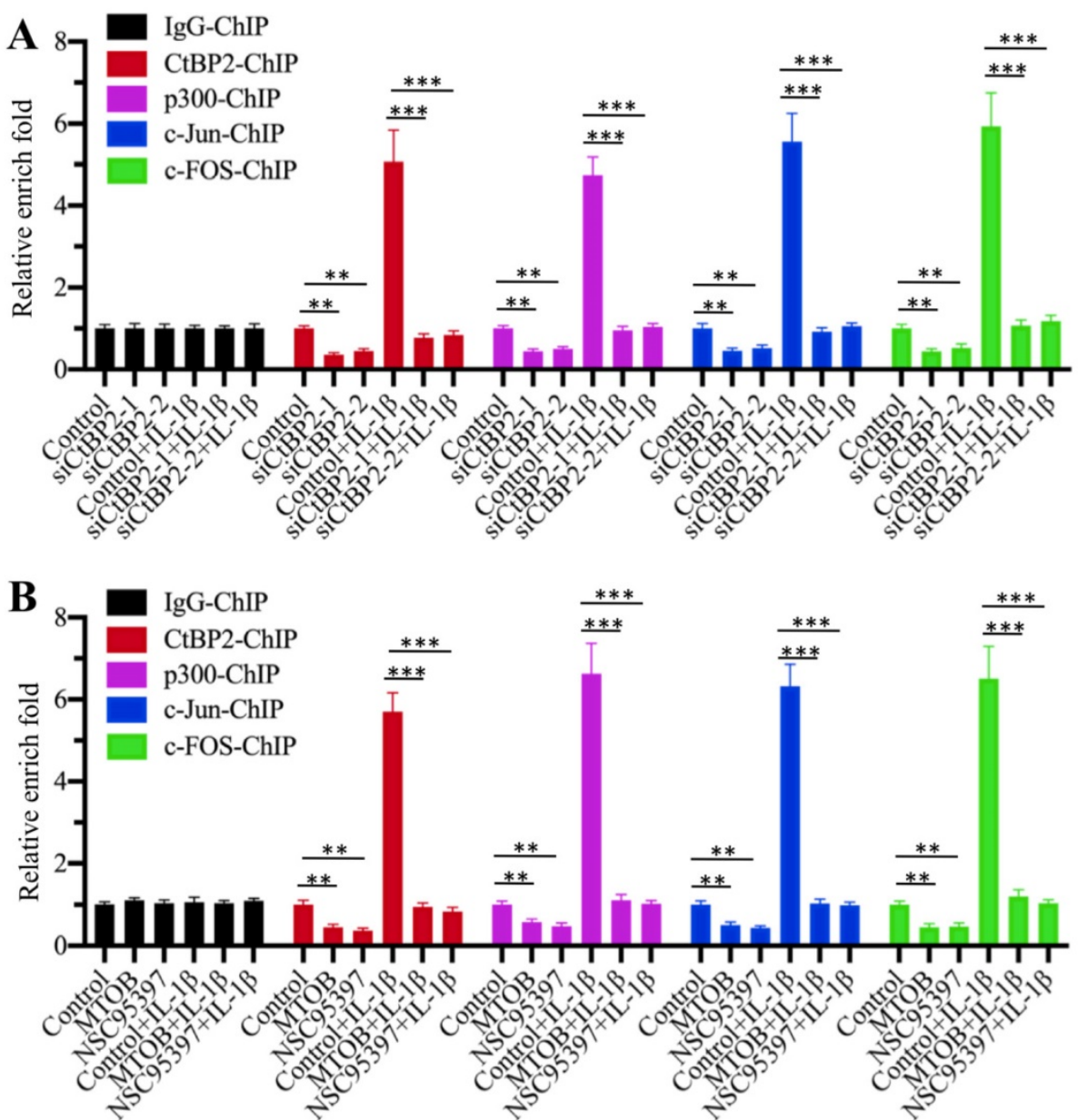

Figure 6. Knockdown or blockage of CtBP2 decreased the occupancies of p300 and AP-1 in the promoter of TGFBI. (A) Knockdown of CtBP2 decreased the occupancies of p300 and AP-1 in the promoter of TGFBI. The RPTEC/TERT1 OAT3 cells were transfected with two independent siRNAs of CtBP2. After incubation for another $48 \mathrm{~h}$, cells were further treated with or without $50 \mathrm{ng} / \mathrm{mL}$ IL-1 $\beta$ for 2 h, followed by ChIP assays using anti-CtBP2, anti-p300, anti-c-Jun and anti-c-FOS to determine their occupancies in the promoter of TGFBI. **P $<0.01$ and $* * * P<0.001$. (B) Blockage of CtBP2 decreased the occupancies of p300 and AP-1 in the promoter of TGFBI. The human RPTEC/TERT1 OAT3 cells were treated with $5 \mu$ M MTOB or NSC95397 for 4 h. Cells were then further treated with or without $50 \mathrm{ng} / \mathrm{mL}$ IL-1 $\beta$ for $2 \mathrm{~h}$. The resulting cells were subjected to ChIP assays using anti-CtBP2, anti-p300, anti-c-Jun and anti-c-FOS to determine their occupancies in the promoter of TGFBI. **P $<0.01$ and ***p $<0.001$. 
Most recently, Zhao and colleagues found that CtBP1 promotes metastasis of breast cancer through activating TGF- $\beta$ signaling [32]. On the other hand, they also found that CtBP1 and ZEB1 (zinc finger E-box binding homeobox 1) are downstream factors of TGF- $\beta$ signaling [32]. Thus, their findings reveal a positive feedback loop between CtBP1-ZEB1 and TGF- $\beta$ signaling [32]. Their results together with our findings suggest that CtBP associates with TFs and may activate TGF- $\beta$ signaling in different biological processes. In addition to this activation, several studies have also revealed that CtBPs can form different transcriptional complexes with p300-Runx2 or HDAC1/2-IRF1 (interferon regulatory factor 1$)$ [23, 33]. In these models, CtBPs function as corepressors, although these complexes also share similar assembly mechanisms in which TFs specifically bind to gene promoters and then recruit histone modification enzymes (p300 or HDAC1/2) and CtBPs $[23,33]$. These results demonstrate that CtBPs can function as both coactivators and corepressors in the regulation of gene expression. However, we still do not understand the underlying mechanism that determines its activation or repression role.

Given that TGF- $\beta$ is a central player in CRF, pharmacological intervention of TGF- $\beta$ is considered as a potential strategy to decrease CRF progression [34]. In our investigation, we found that two AP1 inhibitors, T-5224 and SR11302, and two CtBP inhibitors, MTOB and NSC95397, could impair the assembly of the CtBP2-p300-AP1 complex, thereby reducing the expression of TGFB1 (Figures $2 \mathrm{~F}$ and $5 \mathrm{E}$ ). These results suggest that each component of the CtBP2-p300-AP1 complex can be developed as a therapeutic target in the treatment of CRF. In addition, some p300 inhibitors, such as C646 and garcinol, are also commercially available. We are currently evaluating the in vivo effects of these inhibitors in a CRF mouse model. Another critical issue is that we only found CtBP1 in the Flag-c-Jun-associated complex but not in the Flag-c-FOS-associated complex in our mass spectrometry (Supplementary Table 4 and 5). We did not examine CtBP1 mRNA and protein levels in CRF biopsies, so we cannot conclude if CtBP1 was involved in the regulation of TGFB1 at present.

In summary, we identified that the serum concentration of TGF- $\beta$ was associated with the severity of the CRF stages. CtBP2-mediated transactivation was responsible for TGF- $\beta$ induction in the pathogenesis of CRF. Our results support a model in which CtBP2-p300-AP1 transcriptional complex specifically binds to the promoter of TGFB1 and induces its expression. The mature TGF- $\beta$ is secreted into the extracellular space and functions as a stimulatory signal to activate downstream signaling, including the increase in Smad2 and Smad3 phosphorylation patterns (Figure 7). Pharmacological intervention of TGF- $\beta$ signaling using inhibitors of the CtBP2-p300-AP1 complex may present new therapeutic strategies for CRF.

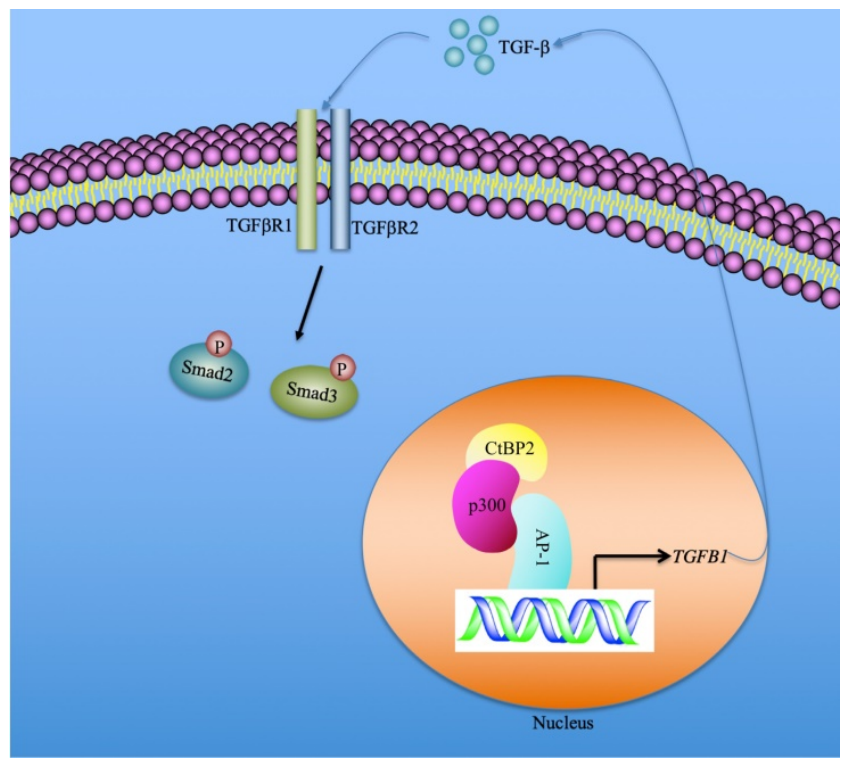

Figure 7. A schematic model of the induction of TGFBI mediated by the CtBP2-p300-API transcriptional complex. In the nucleus, the transcription factor AP-1 specifically binds to the promoter of TGFBI. AP-1 further recruits histone acetyltransferase $p 300$ to assemble a platform of AP1-p300, which further recruits CtBP2 to form a CtBP2-p300-AP1 complex. This complex activates the expression of TGFBI and promotes the production of mature TGF- $\beta$. The mature TGF- $\beta$ is secreted to the extracellular space and functions to stimulate and to initiate TGF- $\beta$ signaling, such as increasing the phosphorylation of Smad2 and Smad3.

\section{Supplementary Material}

Supplementary figures and tables.

http://www.ijbs.com/v16p0204s1.pdf

\section{Acknowledgements}

We thank all participants in this study. We cannot finish this study without their understandings and participation.

\section{Author contributions statement}

Dr. Aimin Zhong and Dr. Ping Zhou designed the research. Dr. Ping Zhou performed most of the experiments. Xiaoxiao Wan, Zhi Chen and Yan Zou performed some of the experiments. Dr. Aimin Zhong and Dr. Ping Zhou analyzed the data, organized the figures and wrote the manuscript. The other authors revised the article.

\section{Ethical approval statement}

The clinical samples were acquired with written informed consent from all of the participants 
following protocols approved by the ethical board of Jiangxi Provincial People's Hospital.

\section{Competing Interests}

The authors have declared that no competing interest exists.

\section{References}

1. Arnold R, Issar T, Krishnan AV, Pussell BA. Neurological complications in chronic kidney disease. JRSM Cardiovasc Dis. 2016;5:2048004016677687.

2. Ruiz-Arenas R, Sierra-Amor R, Seccombe D, Raymondo S, Graziani MS, Panteghini M, et al. A Summary of Worldwide National Activities in Chronic Kidney Disease (CKD) Testing. EJIFCC. 2017;28:302-14.

3. Bailey RA, Wang Y, Zhu V, Rupnow MF. Chronic kidney disease in US adults with type 2 diabetes: an updated national estimate of prevalence based on Kidney Disease: Improving Global Outcomes (KDIGO) staging. BMC Res Notes. 2014;7:415.

4. Ketteler M, Block GA, Evenepoel P, Fukagawa M, Herzog CA, McCann L, et al. Diagnosis, Evaluation, Prevention, and Treatment of Chronic Kidney Disease-Mineral and Bone Disorder: Synopsis of the Kidney Disease: Improving Global Outcomes 2017 Clinical Practice Guideline Update. Ann Intern Med. 2018;168:422-30.

5. Ruiz-Arenas R, Sierra-Amor R, Seccombe D, Raymondo S, Graziani MS, Panteghini M, et al. A Summary of Worldwide National Activities in Chronic Kidney Disease (CKD) Testing. EJIFCC. 2017;28:302-14.

6. Dharmarajan TS, Yoo J, Russell RO, Norkus EP. Chronic kidney disease staging in nursing home and community older adults: does the choice of cockcroft-gault, modification of diet in renal disease study, or the chronic kidney disease epidemiology collaboration initiative equations matter? J Am Med Dir Assoc. 2012;13:151-5.

7. Levey AS, Becker C, Inker LA. Glomerular filtration rate and albuminuria for detection and staging of acute and chronic kidney disease in adults: a systematic review. JAMA. 2015;313:837-46.

8. Henaut L, Grissi M, Brazier F, Assem M, Poirot-Leclercq S, Lenglet G, et al. Cellular and molecular mechanisms associated with ischemic stroke severity in female mice with chronic kidney disease. Sci Rep. 2019;9:6432

9. Gajjala PR, Sanati M, Jankowski J. Cellular and Molecular Mechanisms of Chronic Kidney Disease with Diabetes Mellitus and Cardiovascular Diseases as Its Comorbidities. Front Immunol. 2015:6:340.

10. Glorieux G, Mullen W, Duranton F, Filip S, Gayrard N, Husi H, et al. New insights in molecular mechanisms involved in chronic kidney disease using high-resolution plasma proteome analysis. Nephrol Dial Transplant. 2015;30:1842-52

11. Mihai S, Codrici E, Popescu ID, Enciu AM, Albulescu L, Necula LG, et al. Inflammation-Related Mechanisms in Chronic Kidney Disease Prediction, Progression, and Outcome. J Immunol Res. 2018;2018:2180373.

12. Moradi H, Vaziri ND. Molecular mechanisms of disorders of lipid metabolism in chronic kidney disease. Front Biosci (Landmark Ed). 2018;23:146-61.

13. August P, Sharma V, Ding R, Schwartz JE, Suthanthiran M. Transforming growth factor beta and excess burden of renal disease. Trans Am Clin Climatol Assoc. 2009;120:61-72.

14. Loeffler I, Wolf G. Transforming growth factor-beta and the progression of renal disease. Nephrol Dial Transplant. 2014;29 Suppl 1:i37-i45.

15. Mehta T, Buzkova P, Kizer JR, Djousse L, Chonchol M, Mukamal KJ, et al. Higher plasma transforming growth factor (TGF)-beta is associated with kidney disease in older community dwelling adults. BMC Nephrol. 2017;18:98.

16. Lemon B, Tjian R. Orchestrated response: a symphony of transcription factors for gene control. Genes Dev. 2000;14:2551-69.

17. Smith $\mathrm{E}$, Shilatifard A. The chromatin signaling pathway: diverse mechanisms of recruitment of histone-modifying enzymes and varied biological outcomes. Mol Cell. 2010;40:689-701.

18. Chan HM, La Thangue NB. p300/CBP proteins: HATs for transcriptional bridges and scaffolds. J Cell Sci. 2001;114:2363-73.

19. Vo N, Goodman RH. CREB-binding protein and p300 in transcriptional regulation. J Biol Chem. 2001;276:13505-8.

20. Blevins MA, Huang M, Zhao R. The Role of CtBP1 in Oncogenic Processes and Its Potential as a Therapeutic Target. Mol Cancer Ther. 2017;16:981-90.

21. Sue N, Jack BH, Eaton SA, Pearson RC, Funnell AP, Turner J, et al. Targeted disruption of the basic Kruppel-like factor gene (Klf3) reveals a role in adipogenesis. Mol Cell Biol. 2008;28:3967-78.

22. Wei H, Wang X, Gan B, Urvalek AM, Melkoumian ZK, Guan JL, et al. Sumoylation delimits KLF8 transcriptional activity associated with the cell cycle regulation. J Biol Chem. 2006;281:16664-71.

23. Zhang W, Duan N, Zhang Q, Song T, Li Z, Chen X, et al. The intracellular $\mathrm{NADH}$ level regulates atrophic nonunion pathogenesis through the CtBP2-p300-Runx2 transcriptional complex. Int J Biol Sci. 2018;14:2023-36.

24. Patel J, Baranwal S, Love IM, Patel NJ, Grossman SR, Patel BB. Inhibition of C-terminal binding protein attenuates transcription factor 4 signaling to selectively target colon cancer stem cells. Cell Cycle. 2014;13:3506-18.
25. Paliwal S, Ho N, Parker D, Grossman SR. CtBP2 Promotes Human Cancer Cell Migration by Transcriptional Activation of Tiam1. Genes Cancer. 2012;3:481-90.

26. Jin $\mathrm{W}$, Scotto $\mathrm{KW}$, Hait WN, Yang JM. Involvement of CtBP1 in the transcriptional activation of the MDR1 gene in human multidrug resistant cancer cells. Biochem Pharmacol. 2007;74:851-9.

27. Ray SK, Li HJ, Metzger E, Schule R, Leiter AB. CtBP and associated LSD1 are required for transcriptional activation by NeuroD1 in gastrointestinal endocrine cells. Mol Cell Biol. 2014;34:2308-17.

28. Boxer LD, Barajas B, Tao S, Zhang J, Khavari PA. ZNF750 interacts with KLF4 and RCOR1, KDM1A, and CTBP1/2 chromatin regulators to repress epidermal progenitor genes and induce differentiation genes. Genes Dev. 2014;28:2013-26.

29. Border WA, Noble NA. Cytokines in kidney disease: the role of transforming growth factor-beta. Am J Kidney Dis. 1993;22:105-13.

30. Li C, Xiao XQ, Qian YH, Zhou ZY. The CtBP1-p300-FOXO3a transcriptional complex represses the expression of the apoptotic regulators Bax and Bim in human osteosarcoma cells. J Cell Physiol. 2019.

31. Zhang X, Du K, Lou Z, Ding K, Zhang F, Zhu J, et al. The CtBP1-HDAC1/2-IRF1 transcriptional complex represses the expression of the long noncoding RNA GAS5 in human osteosarcoma cells. Int J Biol Sci. 2019;15:1460-71.

32. Loeffler I, Wolf G. Transforming growth factor-beta and the progression of renal disease. Nephrol Dial Transplant. 2014;29 Suppl 1:i37-i45.

33. Zhao Z, Hao D, Wang L, Li J, Meng Y, Li P, et al. CtBP promotes metastasis of breast cancer through repressing cholesterol and activating TGF-beta signaling. Oncogene. 2019;38:2076-91.

34. Gagliardini E, Benigni A. Therapeutic potential of TGF-beta inhibition in chronic renal failure. Expert Opin Biol Ther. 2007;7:293-304. 